\title{
Repeated use of hormonal emergency contraception by younger women in the UK
}

\author{
Sam Rowlands, MD, MRCGP, MFFP, Medical Director; Hassy Devalia, MRPharmS, Operations Director \\ EPIC, Regeneration House, York Way, London, UK
}

Ross Lawrenson, MD, MRCGP, FAFPHM, Professor of Primary Health Care; John Logie, PhD, Research Fellow, Bernard
Ineichen, PhD, Senior Research Fellow
Postgraduate Medical School, European Institute of Health and Medical Sciences, University of Surrey, Guildford, Surrey, UK

Correspondence: Sam Rowlands, Medical Director, Epic, Regeneration House, York Way, London, N1 0BB, UK. Tel: 020 7713 1118 Fax: 02077131119 email: sam.rowlands@epic-uk.org

(Accepted February $\left.28^{\text {th }}, 2000\right)$

\begin{abstract}
Summary
A cohort of women aged 14-29 in 1993 was identified from the General Practice Research Database and followed up for a period of 4 years. Patient files were searched for evidence of use of emergency contraception and regular contraception. Of the 95007 women, 15105 (16\%) had received emergency contraception during the study period (an average of 5\% per annum). There was a small year on year increase in uptake of emergency contraception between 1994 and 1997. Only 4\% of emergency contraception users received emergency contraception more than twice in any year. More than $70 \%$ of those who had no previous record of use of regular contraception had used regular contraception within 1 year of using emergency contraception. Teenagers were more likely than other age groups to use emergency contraception, to be repeat users of emergency contraception and to fail to start regular contraception after first use of emergency contraception until later in the study period.

These results disprove the notion of widespread repeated use of emergency contraception. They show that provision of an emergency contraception service does not result in failure to initiate regular contraception or abandonment of regular contraception; rather they show many women using regular contraception for the first time after use of emergency contraception.
\end{abstract}

\section{Key words}

attitude of health personnel, contraception behaviour, drug prescriptions, family planning, family practice, post-coital contraceptives, physician's practice patterns, sex behaviour

\section{Key message points}

- Repeated use of emergency contraception was rare in this study.

- The majority of women in their 20 s had used regular contraception before their first use of emergency contraception.

- Both use and repeat use of emergency contraception were more common in teenagers.

- The small minority using emergency contraception repeatedly is no less likely to use regular contraception before first use of emergency contraception or to start regular contraception soon after first use of emergency contraception.

\section{Introduction}

It is now 30 years since hormonal emergency contraception (EC) became available in the $\mathrm{UK}^{1}$ and 15 years since the launch of Schering PC4. Despite widespread availability through GPs, family planning clinics, accident and emergency departments and elsewhere, and knowledge among the public as high as $94 \%,{ }^{2}$ uptake of EC remains relatively low. It is estimated that 810000 prescriptions for EC were issued in the UK in $1998 .^{3}$ Ignoring other products and methods of EC, and ignoring women sterilised or infertile, of 12.2 million women at risk there was the equivalent of only one treatment for 1 in 15 women during 1998. Could it be that health professionals' concerns are still acting as a barrier to access to EC?

There have, in the past, been safety concerns, but these have now been shown to be groundless. ${ }^{4}$ For instance, because the oestrogen exposure is so transient, there is no detectable increased risk of deep venous thrombosis or pulmonary embolism following use of Schering PC4. ${ }^{5}$ There have also been concerns that EC is abortifacient; EC does not cause loss of established pregnancy but works by preventing implantation., 6

There is a genuine concern that the efficacy of EC is poor compared with regular contraception (RC) and repeated use disrupts the menstrual cycle. However, there will always be episodes of unprotected intercourse because of the nature of human relationships and impulsive sexual behaviour. Firsttime ever intercourse is notorious for lack of contraceptive protection $;^{8}$ similar behaviour is seen in couples initiating a new relationship or who are reunited with an existing partner or ex-partner. ${ }^{9}$ More women in the 16-24 age group than in older age groups report being drunk as a contributory factor to losing their virginity. ${ }^{8}$ For teenagers, poor skills in talking about sex, negotiating relationships and taking responsibility for the outcomes, together with lack in confidence and discipline in using condoms, are all factors in non-use of contraception. ${ }^{10}$ Most UK family planning professionals would agree that use of EC is preferable to abortion.

Some women already find seeking emergency contraception an embarrassing and even humiliating experience. Some cannot face attending at all. Judgmental or inept attitudes by some health professionals compound the situation. 11,12

EC is not officially recommended for repeated use, ${ }^{13}$ but any anxiety on behalf of clinicians about the safety of repeated use is not justified. ${ }^{14}$ Some health professionals have taken a disapproving moral view on repeated use of EC, rather like that on repeat abortion. ${ }^{15}$ We know that $26 \%$ of women having terminations in England and Wales have had one or more previous abortion ${ }^{16}$ and that this has increased from $8 \%$ in $1977 .{ }^{17}$ It is likely that fewer health professionals are now taking a judgmental attitude to repeated use of EC while continuing to emphasise to patients the better option of employing a regular method of contraception. There is evidence that GPs are less 
judgmental than accident and emergency departments, as reported by senior nursing staff in terms of whether they would be prepared to prescribe for women who had been treated within the previous 3 months. ${ }^{18}$ However, in a qualitative study of GPs' attitudes to EC, many of the 76 respondents drew an arbitrary line and gave a maximum number of times they would provide EC. ${ }^{19}$ Only one GP would routinely tell a woman when EC was being given that it was all right to use EC again.

It is difficult to know whether the negative attitudes shown by health professionals are due to genuine moral/medical concerns, or to the fact that (especially in general practice) a request for EC is liable to disrupt appointment systems. Nevertheless, many GPs remain concerned about repeated use of EC and may communicate this disquiet to their patients. There remains a feeling among some health professionals that EC may be 'abused' by women, with reliance on EC instead of organising RC.

It has clearly been shown that these concerns are unfounded. In a classic study in which women were randomly allocated to advance (i.e. before a possible episode of unprotected intercourse) prescription of the Yuzpe regimen or information about its availability only, ${ }^{20}$ women in the treatment group were significantly more likely than controls to use EC once, but not more likely to use EC more than once. Eighty-nine percent of women in the treatment group reported a year later that their use of RC was unaffected, and similar numbers in each group switched from using barrier methods to using more reliable methods of contraception.

Prescribing of EC is predominantly to a young age group. GP database studies show a peak prescribing rate of Schering PC4 at age 18 with very little prescribing over age 26 in UK general practice. ${ }^{21}$ Research from one general practice has shown that 59/373 (16\%) of girls aged 15-19 had consulted for emergency contraception: 40 once only, 11 twice and eight on three occasions. ${ }^{22}$ Family planning clinic statistics show peak use in the 16-19 age group. ${ }^{3}$

This study set out to establish how common repeated use of EC is in younger women in general practice, and to relate this to subsequent establishment on RC.

\section{Method}

A cohort of all permanently registered females aged 14-29, inclusive, in 1993 was identified from the General Practice Research Database. The General Practice Research Database contains the records of over seven million patients in the UK and has been validated to contain more than $90 \%$ of all prescriptions issued. ${ }^{23}$ Contributing practices are widely distributed throughout all four countries of the UK; rural and urban locations are represented. A cohort study was conducted from 1994-1997, inclusive. Data on the patients in the cohort were required to be up to the quality standard set by the Office for National Statistics throughout the whole study period. Files were searched for evidence of prior sexual activity as evidenced by previous pregnancies, previous use of EC and previous use of RC. Prescription codes for Schering PC4 and OXMIS codes for EC were searched for during the study period (we did not investigate use of postcoital IUDs). We also searched during the study period for prescription codes for all methods of RC together with OXMIS codes for specific methods of contraception, including barrier methods and attendance for contraceptive care. We defined repeat EC use as more than one entry for EC use in a set of records in any one of the 4 years of the study.

Relationships between EC use and the personal characteristics of women within the study population were examined using chi-square tests of association considering age (partitioned into the age groups 14-19, 20-24 and 25-29 years), smoking status, evidence of prior sexual activity and records of RC. For women with records of EC, repeat use and the numbers of EC records were examined using MannWhitney and Kruskal-Wallis tests where appropriate.

\section{Results}

Data were extracted from a total of 357 practices. There were data for 95007 women of whom 15105 (15.9\%) received EC during the study period. The results are summarised in Table 1. Women aged 14-19 were more likely $(21.1 \%)$ and women aged $25-29$ less likely $(11.5 \%)$ to have records of EC use $\left(\chi_{2}^{2}=942.41, \mathrm{p}<0.0001\right)$.

Table 2 shows use for all three age groups for each year of the study split into EC use once, twice and more than twice. This shows that EC use more than twice in any 1 year is rare: only $4 \%$ of EC users received EC more than twice in any year. Repeat use taken over the entire 4-year study period was also rare: use twice was $2.9 \%$ of women, use 3 times was $1.0 \%$ and use four or more times was $0.8 \%$. Repeat users were most likely to come from the 14-19 age group $\left(\chi_{2}^{2}=48.07, \mathrm{p}<0.0001\right)$. The maximum number of EC records for any individual ranged between seven and 12 within a single year, with an overall maximum of 17 records during all 4 years of the study.

For all groups, use of EC increased during the 4 years of the study and this was only due in part to an aging effect. Accounting for age (by redefining the age groups to consider age in the year in question, rather than the cohorts defined from age in 1993), between 1994 and 1997 the percentage of women with records of EC use within a single year rose from $4.2 \%$ to $6.5 \%$ for women aged $20-24$ and from $2.9 \%$ to $4.6 \%$ for women aged $25-29$. Changes in EC use between years could not be fully considered in the 14-19 age group because data on the youngest women were only available for the first year. Nevertheless, for 18 and 19 year olds (the ages most likely to use EC) where data were available in all 4 years, the proportion of women with EC records rose from $6.0 \%$ in 1994 to $8.4 \%$ in 1997 , with a peak of $8.6 \%$ in 1996 .

Use of RC during the study period was lowest in the $14-19$ age group $(63.0 \%)$ and highest in the 20-24 age group $(77.5 \%)$. There was little evidence that the proportion of women using RC within each age group changed between years.

Evidence of prior use of RC $\left(\chi_{2}^{2}=26256.31\right.$, $\mathrm{p}<0.0001)$, prior pregnancy $\left(\chi_{2}^{2}=17051.70, \mathrm{p}<0.0001\right)$ and prior sexual activity from all three indicators combined $\left(\chi_{2}^{2}=31111.92, \mathrm{p}<0.0001\right)$ was more likely with increasing age. This did not apply with respect to prior use of EC, for which women aged 20-24 had significantly more

Table 1 Characteristics of cohort (Percentages unless otherwise specified)

\begin{tabular}{lcccc}
\hline Age group & $14-19$ & $20-24$ & $25-29$ & $\mathbf{1 4 - 2 9}$ \\
Women in age group (numbers) & 33107 & 27626 & 34274 & $\mathbf{9 5 0 0 7}$ \\
Prior pregnancy (1) & 5.0 & 29.9 & 50.5 & $\mathbf{2 8 . 6}$ \\
Prior EC use (2) & 4.1 & 10.8 & 6.5 & $\mathbf{6 . 9}$ \\
Prior RC use (3) & 19.7 & 72.6 & 76.0 & $\mathbf{5 5 . 4}$ \\
Prior sexual activity (1+2+3) & 22.4 & 76.9 & 84.0 & $\mathbf{6 0 . 5}$ \\
EC use during study (4) & 21.1 & 16.3 & 11.5 & $\mathbf{1 5 . 9}$ \\
RC use during study (5) & 63.0 & 77.5 & 70.9 & $\mathbf{7 0 . 1}$ \\
Total contraceptive use during study (4+5) & 65.4 & 78.8 & 72.3 & $\mathbf{7 1 . 8}$ \\
Total contraceptive use ever $(2+3+4+5)$ & 66.8 & 86.0 & 85.2 & $\mathbf{7 9 . 0}$ \\
\hline
\end{tabular}

$\mathrm{EC}=$ Emergency contraception

$\mathrm{RC}=$ Regular contraception 
records than expected $\left(\chi_{2}^{2}=1062.23, \mathrm{p}<0.0001\right)$. Within all age groups there was a positive association between use of EC and prior sexual activity $\left(\chi_{2}^{2} \geq 92.24, \mathrm{p}<0.0001\right)$. EC users with records of prior sexual activity also appeared more likely to be repeat users, having significantly more EC records during the full 4-year period (M-W $\mathrm{Z} \leq-2.95$, $\mathrm{p}<0.0001$ for all age-groups).

Fifty-three percent of all records contained details of subjects' smoking status. Of these women, $37.3 \%$ were current smokers. For all age groups, smokers were more likely to have used EC than non-smokers $\left(\chi_{1}^{2} \geq 63.78\right.$, $\mathrm{p}<0.0001)$. Although a significant association was found between smoking and age $\left(\chi_{2}^{2}=73.95, \mathrm{p}<0.0001\right)$, this would not explain the higher frequency of EC records in the youngest age group. The 14-19 age group contained a marginally lower proportion of smokers than all ages (35.3\% for ages $14-19$, compared to $39.9 \%$ and $36.9 \%$ for the 20-24, and 25-29 year age groups, respectively). Considering only women with one or more record of EC use within the year, smokers were no more likely to be repeat users of EC than non-smokers in any of the 4 years of the study (M-W $Z \geq-1.58, p \geq 0.115$ in all cases). Over the full 4-year period, however, among EC users in the 14-19 age group smokers did have significantly more EC records $(\mathrm{M}-\mathrm{W} \mathrm{Z}=-2.13, \mathrm{p}=0.03)$. No significant differences were apparent in the total number of records for EC users between smokers and non-smokers for women aged 20-24 years (M-W Z $=-1.24, \mathrm{p}=0.22)$, or 24-29 years (M-W Z $=-1.89, \mathrm{p}=0.06)$.

The temporal relationship between first use of EC and first use of RC was examined by looking at the 6-month period when EC was first used and ascertaining whether first use of RC occurred before, during or after the same 6 -month period. Overall, only $8.8 \%$ of women had records of EC use prior to their first record of RC. The first records of both EC and RC fell within the same 6-month period for a further $18.9 \%$ of women. In $72.3 \%$ of cases, the first EC record was found in a 6-month period after that with the first RC record. Significant differences existed between age groups, however, with the 14-19 age group showing an entirely different pattern of use compared with the two older age groups, which had very similar patterns $\left(\chi^{2}{ }_{1}=2128.67, \mathrm{p}<0.0001\right)$. The $14-19$ age group were much less likely than the $20-29$ s to have a record of previous use of RC (53.2\% v 89.7\%), and were much more likely to start use of regular contraception in the same 6-month period as their first EC use than the others $(31.6 \%$ $\mathrm{v} 7.3 \%$ ). They were also more likely to fail to start RC until later in the study period than the others $(15.2 \% \mathrm{v} 3.0 \%)$. Of the 1077 women with a record of EC use between 1994 and 1997 who failed to start RC at the time of their first EC record and who had no previous record of $\mathrm{RC}, 49.9 \%$ subsequently started RC within the following 6 months, and $71.5 \%$ had started within the following year. No differences were found in the timing of the start of RC between single and repeat EC users $\left(\chi_{2}^{2} \leq 3.60, \mathrm{p} \geq 0.17\right.$ for all age groups). However, women who had their first record of EC before their first $\mathrm{RC}$ record were more likely to be repeat EC users $\left(\chi_{2}^{2} \geq 35.79, \mathrm{p}<0.0001\right.$ for all age groups). This difference was most pronounced within the youngest age group, where repeat users were almost twice as likely to have used EC prior to $\mathrm{RC}$, than women with only a single EC record $(22.8 \%$ v $12.9 \%$ of EC users). For all age groups, women who failed to start RC until after the 6-month period of their first EC use had more EC records over the entire study duration ( $\mathrm{K}-\mathrm{W} \chi^{2}{ }_{2} \geq 2.49, \mathrm{p}<0.0001$ for all age groups). Women who had used $\mathrm{RC}$ at any time tended to have more records of EC use. For all age groups, the total number of occasions of EC use over the entire study period was greater for EC users who had at least one entry for RC use than for those without $(\mathrm{M}-\mathrm{W} \mathrm{Z} \leq-4.62, \mathrm{p}<0.001$ for all age groups).

\section{Discussion}

Possible sources of inaccuracy of the data used in this study must first be considered. It has to be accepted that information about contraception in women in this age group may be missing from the GP records. Permission is not always given for a letter to go from a family planning clinic to the GP, especially by the very young. There are no follow-up studies on switching of use from family planning clinic services to GP services and vice versa. There is differential use of services according to age group: whereas $76 \%$ of contraceptive advice is obtained from general practice overall, ${ }^{24}$ only $29 \%$ of such advice is obtained from general practice by girls aged under $16 .{ }^{10}$ The extent of use of accident and emergency departments for access to EC is unknown and notification of GPs of treatment from this service outlet may not always occur. The General Household Survey indicates that on $62 \%$ of the occasions

Table 2 Number of times emergency contraception used by year and age group (Numbers/percentages)

\begin{tabular}{|c|c|c|c|c|c|c|c|c|c|}
\hline & & \multicolumn{2}{|c|}{ 14-19 years } & \multicolumn{2}{|c|}{ 20-24 years } & \multicolumn{2}{|c|}{$25-29$ years } & \multicolumn{2}{|c|}{ 14-29 years } \\
\hline & & $\mathrm{N}$ & $\%$ & $\mathrm{~N}$ & $\%$ & $\mathrm{~N}$ & $\%$ & $\mathrm{~N}$ & $\%$ \\
\hline \multirow[t]{3}{*}{1994} & 0 & 31801 & 96.1 & 26475 & 95.8 & 33293 & 97.1 & 91569 & 96.4 \\
\hline & 1 & 1106 & 3.3 & 1005 & 3.6 & 872 & 2.5 & 2983 & 3.1 \\
\hline & 2 & 135 & 0.4 & 109 & 0.4 & 83 & 0.2 & 327 & 0.3 \\
\hline \multicolumn{2}{|c|}{3 or more } & 65 & 0.2 & 37 & 0.1 & 26 & 0.1 & 128 & 0.1 \\
\hline \multirow[t]{3}{*}{1995} & 0 & 31160 & 94.1 & 26260 & 95.1 & 33027 & 96.4 & 90447 & 95.2 \\
\hline & 1 & 1646 & 5.0 & 1186 & 4.3 & 1127 & 3.3 & 3959 & 4.2 \\
\hline & 2 & 217 & 0.7 & 121 & 0.4 & 81 & 0.2 & 419 & 0.4 \\
\hline \multicolumn{2}{|c|}{3 or more } & 84 & 0.3 & 59 & 0.2 & 39 & 0.1 & 182 & 0.2 \\
\hline \multirow[t]{3}{*}{1996} & 0 & 30570 & 92.3 & 26055 & 94.3 & 32951 & 96.1 & 89576 & 94.3 \\
\hline & 1 & 2077 & 6.3 & 1358 & 4.9 & 1146 & 3.3 & 4581 & 4.8 \\
\hline & 2 & 322 & 1.0 & 146 & 0.5 & 126 & 0.4 & 594 & 0.6 \\
\hline \multicolumn{2}{|c|}{3 or more } & 138 & 0.4 & 67 & 0.2 & 51 & 0.1 & 256 & 0.3 \\
\hline \multirow[t]{3}{*}{1997} & 0 & 30577 & 92.4 & 26096 & 94.5 & 33036 & 96.4 & 89709 & 94.4 \\
\hline & 1 & 2112 & 6.4 & 1324 & 4.8 & 1088 & 3.2 & 4524 & 4.8 \\
\hline & 2 & 298 & 0.9 & 132 & 0.5 & 112 & 0.3 & 542 & 0.6 \\
\hline \multicolumn{2}{|c|}{3 or more } & 120 & 0.4 & 74 & 0.3 & 38 & 0.1 & 232 & 0.2 \\
\hline
\end{tabular}


women aged 16-29 had sought EC they had used their GP for the service. ${ }^{25}$ We have no reason to suppose that repeated use of EC would be more or less likely with a particular service outlet. The rate of use of family planning clinics by teenagers has been increasing steadily until last year; each successive year since 1985 for girls under 16 and since 1988 for women aged 16-19. ${ }^{3}$ The first record of contraceptive use may well be more likely to be held by a family planning clinic than by the GP, especially in recent years. For these reasons, our study is likely to have underestimated contraceptive use in teenagers. Another inadequacy of the data, as with most general practice data, is the lack of information on condom use which gives a sizeable underestimate of use of RC.

Contraceptive uptake during the study was more than $70 \%$ and contraceptive use ever was almost $80 \%$. These figures are high and indicate comprehensive data recording by GPs contributing to the General Practice Research Database. Figures from a 1995 national cross sectional survey for current use of any method of contraception in the 16-29 age group show 62\% uptake (personal communication, General Household Survey 1999).

Our figure for the extent of use of EC during a 4-year period of $15.9 \%$ is similar to the national figure for 1995 of $12 \%$ use over a 2-year period for women aged 16-29 who were not sterilised, and whose partners were not sterilised (personal communication, General Household Survey, 1999). It is also similar to the figure of $14.3 \%$ ever use found in a survey of women aged 18-47 in Grampian. ${ }^{2}$ Obviously surveys may underestimate prevalence because of recall bias.

General practice records show that repeated use of EC is rare. The result is similar to that of a survey of Finnish girls aged 14-17: among those who had ever used EC, only $4.9 \%$ had used EC more than twice. ${ }^{26}$ Use of EC is predominantly by teenagers who are likely to be initiating sexual relationships. The majority of women in their 20 s had used $\mathrm{RC}$ before their first use of EC and were probably using EC where there had been a condom mishap or forgotten pills or when unprotected intercourse occurred on being reunited with a partner or after having changed partners. On the other hand, teenagers' use of EC may often have been their first experience of accessing contraceptive services; the majority was subsequently using RC soon afterwards.

Both use and repeat use of EC was more common in the 14-19 age group. This fits with exploratory sexual behaviour in teenagers. The small increase in use of EC year on year probably reflects improvement in access to, and public knowledge of, EC during the study period. The fact that prior use of EC by women aged 25-29 was less than in those aged 20-24 probably indicates poorer public knowledge existing a decade previously when the former group were at their peak use of the method.

Highest use of RC was in the 20-24 age group. This fits with this age group having the highest abortion rate, indicating stronger motivation to avoid having children. Use of RC declines in the 25-29 age group which is known to have the highest birth (fertility) rate.

Prior sexual activity increases with age as one would expect. The National Survey of Sexual Attitudes and Lifestyles shows a large difference in the prevalence of never having had a heterosexual partner with age: $21 \%$ in the 16-24 age group and only $2 \%$ in the $25-34$ age group. ${ }^{8}$ We find the level of $84 \%$ (Table 1) remarkably high, indicating nearly complete data on sexual activity from electronic sources

This study, and another by us not yet published of women aged 10-44 years prescribed PC4 between 1992 and 1998, provide a baseline measurement of use of EC in UK general practice. Major shifts in EC service provision in the UK are likely in the next few years with the licensing of progestogen-only contraception ${ }^{27}$ and evidence that efficacy is greater if EC is administered in the first 12-24 hours. $^{28}$ There is already access via nurses ${ }^{29}$ and pharmacists $^{30}$ working to protocols, and prescribing EC in advance has now begun. ${ }^{31,32}$ Prescribing by nurses ${ }^{33}$ and pharmacists $^{34}$ is likely soon, and there is the inevitable progression to over-the-counter availability as already exists in France. ${ }^{35}$

\section{Conclusions}

We conclude that the small minority using EC repeatedly are no less likely to use RC before first use of EC, or to start $\mathrm{RC}$ soon after first use of EC, than are those who use EC only once in any 1 year. It is unfortunate that some health professionals make arbitrary judgements about how many EC uses a year are acceptable to them. We know from studies in Britain, ${ }^{12,18}$ the $\mathrm{US}^{36}$ and Zimabwe ${ }^{37}$ that women seeking EC often receive a less than sympathetic welcome, and are given incorrect information.

The results of our study disprove the notion of widespread repeated use of EC and hopefully will reassure GPs and others that provision of an EC service does not result in failure to initiate $\mathrm{RC}$ or abandonment of RC. They support widespread access to EC as an integral component of a comprehensive family planning service.

\section{Acknowledgement}

Tom Gebsattel performed the computer programming.

Statements on funding and competing interests

Funding. None.

Competing interests. None.

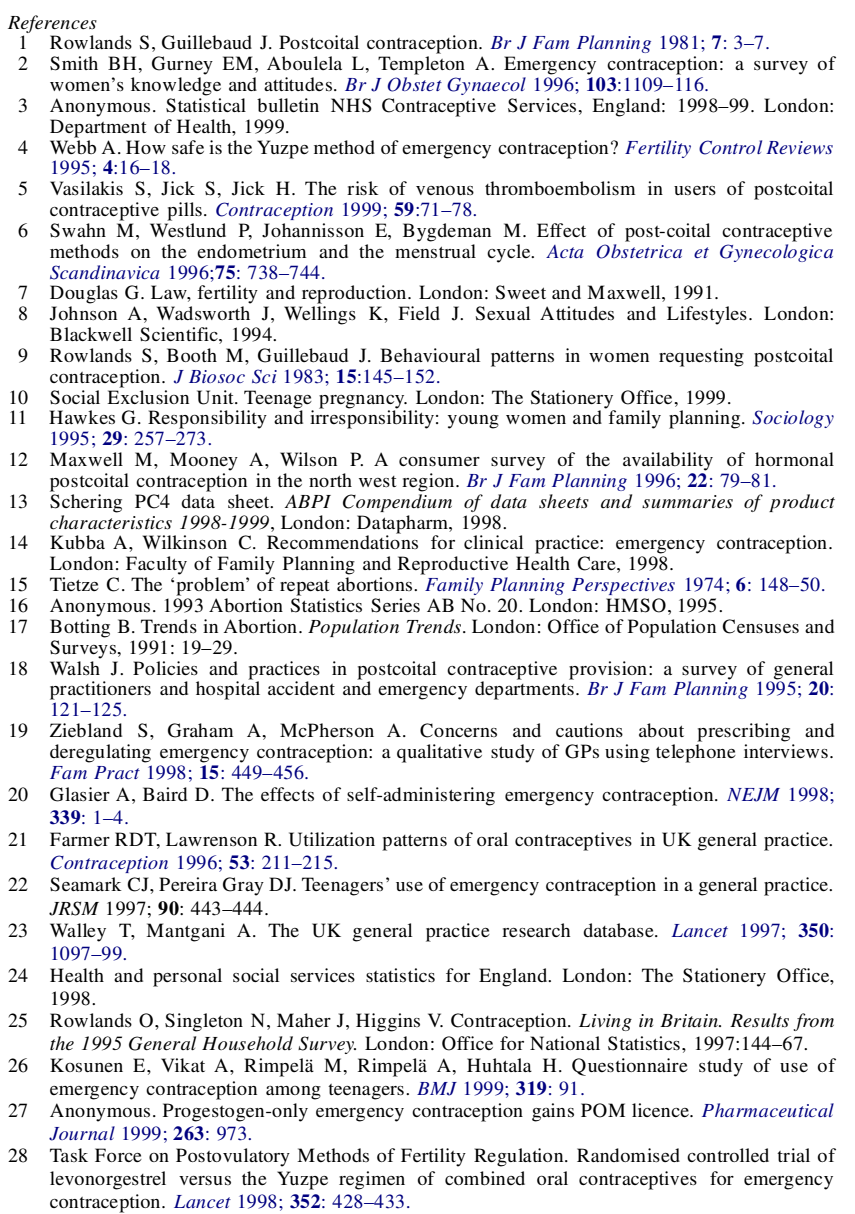

Rowlands S, Guillebaud J. Postcoital contraception. Br J Fam Planning 1981; 7: 3-7. on the endometrium Blackwell Scientific, 1994

ery Office, 1999

M. Wilson P. A consumer survey of the availability of hormona .

PC4 data sheet. ABPI Compendium of data sheets and summaries of product

.

. Botting B. Trends in Ation Statistion

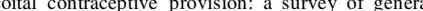
$21-125$

Ziebland S, Graham A, McPherson A. Concerns and cautions about prescribing an

Glasier A, Baird D. The effects of self-administering emergency contraception. NEJM 1998; 339: $1-4$,

(n) RSM 1997; 90: 443-444.

1097-99.

Health and personal social services statistics for England. London: The Stationery Office, Rowlands O, Singleton N, Maher J, Higgins V. Contraception. Living in Britain. Results from the 1995 General Household Survey. London: Office for National Statistics, 1997:144-67. emergency contraception among teenagers. BMJ 1999; 319: 91 .

Anonymous. Progestogen-only emergency contraception gains POM licence. Pharmaceutical Task Force on Postovulatory Methods of Fertility Regulation. Randomised controlled trial o contraception. Lancet 1998; 352: 428-433. 
29 Brittain D. Establishing an educational programme for nurses to supply emergency hormonal contraception (combined method) to protocol. Br J Fam Planning 1999; 25: 118-121.

O'Brien K, Gray N. Supplying emergency hormonal contraception in Manchester under a group prescribing protocol. Pharmaceutical Journal 2000; 264: 518-519.

1 British Pregnancy Advisory Service. Press release: Emergency contraception ... before the emergency. 8th July1999.

32 Christie B. Project makes emergency pill more available. BMJ 1999; 319: 661

33 Anonymous. Review of prescribing, supply and administration of medicines: final report. London: Department of Health, 1999.
34 Anonymous. Parliamentary group wants pharmacies to supply emergency contraception Pharmaceutical Journal 2000; 264: 354 .

35 Anonymous. French pharmacists can supply emergency contraception. Pharmaceutical Journal 1999; 263: 563

36 Gold M, Schein A, Coupey SM. Emergency contraception: a national survey of adolescent health experts. Family Planning Perspectives 1997: 29:15-9 and 24.

37 Rutgers RAK,Verkuyl DAA. Please help, our condom tore last night. S Afr Med J 1998; 88 $143-145$

品

c กิ 